\title{
Caso clínico: Sialolito gigante en el conducto de Wharton
}

\section{Case report: Giant sialolite in the Wharton duct}

Recibido: 2017/04/15. Aceptado: 2017/06/20. Publicado: 2017/09/01

\section{Juan Diego Salazar ${ }^{1}$ \\ Dr. Fernando Sandoval V. ${ }^{2}$ \\ Estefanía Carcelén ${ }^{3}$ \\ Fernando Sandoval Portilla ${ }^{4}$}

1 Universidad San Francisco de Quito, Colegio de Ciencias de la Salud, Escuela de Odontología, Clínica Odontológica, Campus Cumbayá, oficina CC 100, casilla postal 17-1200-841. Quito-Ecuador.

Correo electrónico: juandieguito87@aol.com

2 Universidad San Francisco de Quito, Colegio de Ciencias de la Salud, Escuela de Odontología, Clínica Odontológica, Campus Cumbayá, oficina CC 100, casilla postal 17-1200-841. Quito-Ecuador.

Correo electrónico: fsandoval@usfq.edu.ec

${ }^{3}$ Universidad San Francisco de Quito, Colegio de Ciencias de la Salud, Escuela de Odontología, Clínica Odontológica, Campus Cumbayá, oficina CC 100, casilla postal 17-1200-841. Quito-Ecuador.

Correo electrónico: estefyp20@hotmail.com

4 Universidad San Francisco de Quito, Colegio de Ciencias de la Salud, Escuela de Odontología, Clínica Odontológica, Campus Cumbayá, oficina CC 100, casilla postal 17-1200-841. Quito-Ecuador.

Correo electrónico: fjose28@hotmail.com 


\section{Resumen}

La sialolitiasis es una afección frecuente de las glándulas salivales mayores, con una frecuencia de aparición del 1.2\% en la población mundial, ocurre con mayor frecuencia en la glándula submandibular. Esta se produce debido a la obstrucción de la glándula salival o del conducto de la misma debido a un sialolito. Este cálculo se desarrolla como resultado de un estancamiento de saliva, este proporciona un ambiente idóneo para la deposición de sales y otras sustancias orgánicas formando un cálculo. El tamaño de los sialolitos frecuentemente es de 1 a $10 \mathrm{~mm}$ y en raras ocasiones se puede apreciar sialolitos gigantes, estos pueden llegan a medir más de $15 \mathrm{~mm}$ y usualmente se localizan en el conducto de Wharton. En este artículo se reporta un caso de un sialolito de $25 \mathrm{~mm}$ de diámetro que se localizaba en el conducto de Wharton, el cual no presentaba sintomatología y fue descubierto por un examen de rutina, y el tratamiento que se eligió fue la remoción por vía intraoral.

Palabras clave: Sialolito, sialolitiasis, glándula submandibular, conducto de Wharton.

\section{Abstract}

Sialolithiasis is a frequent condition of the major salivary glands, with a frequency of occurrence of $1.2 \%$ in the world population, occurs more frequently in the submandibular gland. This occurs due to obstruction of the salivary gland or its excretory duct due to a calculation. This salivary calculi develop as a result of saliva stagnation; this provides an ideal environment for the deposition of salts and other organic substances forming a sialolith. The size of sialoliths is often 1 to $10 \mathrm{~mm}$ and in rare cases giant sialoliths can be seen, this can reach more than $15 \mathrm{~mm}$ and are usually located in the Wharton's duct. This article reports a case of a $25 \mathrm{~mm}$ diameter sialolite located in the Wharton's duct, which had no symptoms and was discovered by a routine examination, and the treatment chosen was intraoral removal.

Key words: Sialolith, sialolithiasis, submandibular gland, Wharton's duct. 


\section{Introducción}

La sialolitiasis es la patología más común de las glándulas salivales, es una condición caracterizada por obstrucción de una glándula salival o de su conducto excretor debido a cálculos 1,2. A nivel mundial los hombres son afectados en una relación de 2:1 en relación a las mujeres, además puede ocurrir a cualquier edad pero el pico de incidencia se presenta en la tercera y cuarta década de la vida 3 .

Se estima una frecuencia del $1.2 \%$ en la población mundial, la mayoría de los cálculos de las glándulas salivares ocurren en la glándula submaxilar se estima un porcentaje del $80-95 \%$ mientras que solo del $5-20 \%$ ocurren en la parótida y el $1-2 \%$ ocurren en las glándula sublingual y las glándulas salivales menores, la glándula submandibular es la más frecuentemente afectada debido a la longitud y angulación del conducto de Wharton 4,3. Los sialolitos localizados en los conductos son usualmente alargados, mientras que los que se localizan en la glándula son redondos u ovalados. El tamaño de los cálculos salivales puede variar desde menos de $1 \mathrm{~mm}$ a unos pocos centímetros en diámetro. La mayoría de los cálculos llegan a medir menos de $10 \mathrm{~cm}(88 \%)$, solo el $7,6 \%$ son más largos de $15 \mathrm{~mm}$ 3,2,4. Los sialolitos están formados de fosfato de calcio con pequeñas cantidades de carbonato en forma de hidroxiapatita, magnesio, potasio y amoniaco ${ }^{1}$.

La sialolitiasis puede ser difícil de diagnosticar debido a que el sialolito no siempre es visible y a la variabilidad de los síntomas, clínicamente se caracteriza por dolor local, inflamación, disminución del flujo salival, limitación de la apertura bucal y contenido purulento 5,6. Los sialolitos de pequeño tamaño pueden ser expulsados espontáneamente mediante la estimulación del flujo salival, aplicación de masajes locales o la administración de sialogogos; en los sialolitos de mayor tamaño en tratamiento consiste en la remoción quirúrgica ${ }^{6}$.
El objetivo de este estudio es reportar los aspectos clínicos de un caso de sialolito gigante en el conducto de Wharton y así como describir las posibles causas, su incidencia y opciones de tratamiento.

\section{Caso clínico}

Paciente masculino de 64 años de edad, asintomático, se presenta en la consulta para valorar la posibilidad de colocación de implantes dentales. En la historia detallada el paciente presentaba una zona edéntula a nivel de la región de órgano dental 36-37, además de la presencia de una inflamación a nivel del piso de la boca del lado izquierdo (Figura 1).

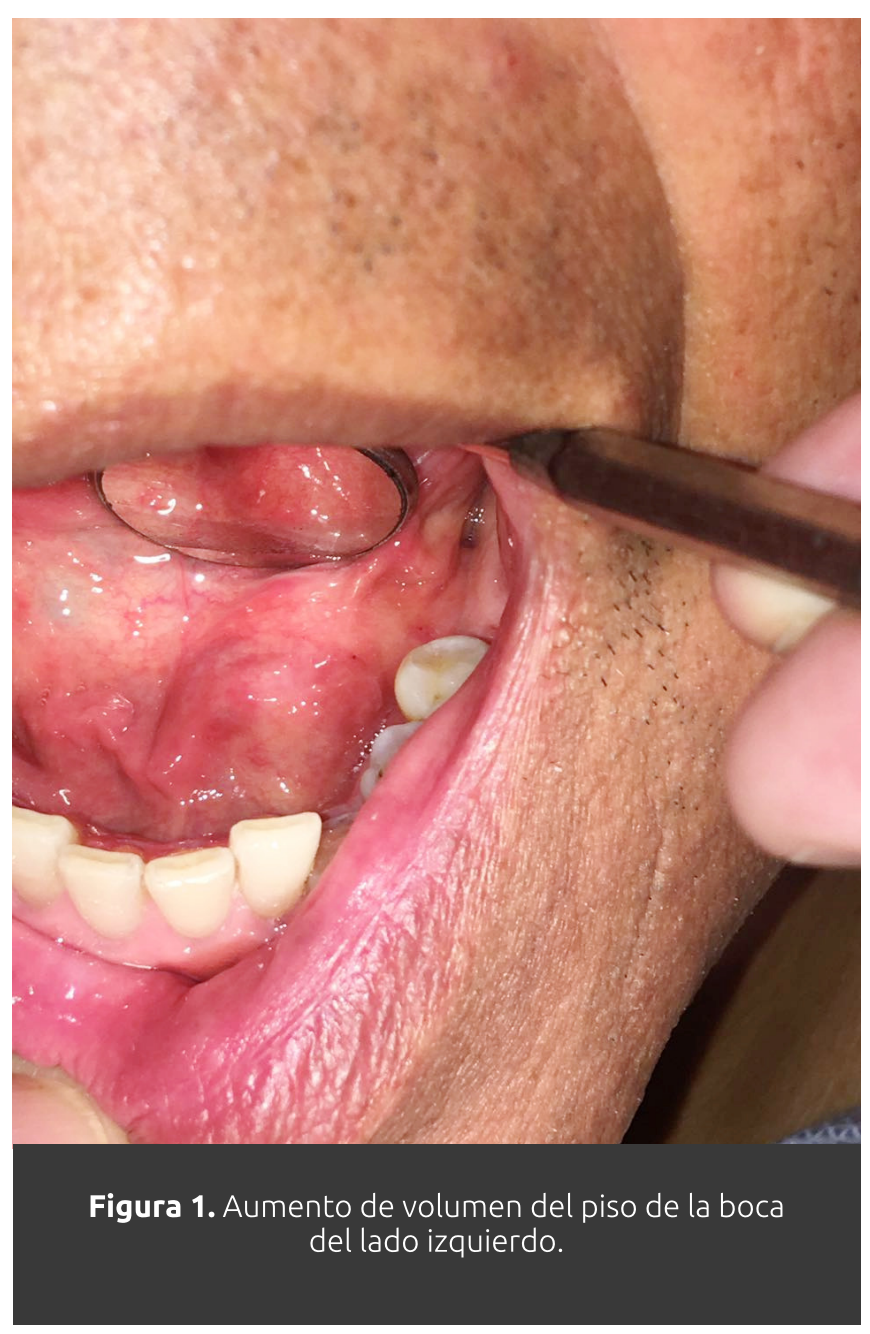

A la examinación intraoral se apreció un aumento de volumen circunscrito de aproximadamente $3 \mathrm{~cm}$ de diámetro en el piso de la boca a nivel de las piezas 32-33-34-35. La mucosa que lo rodeaba 

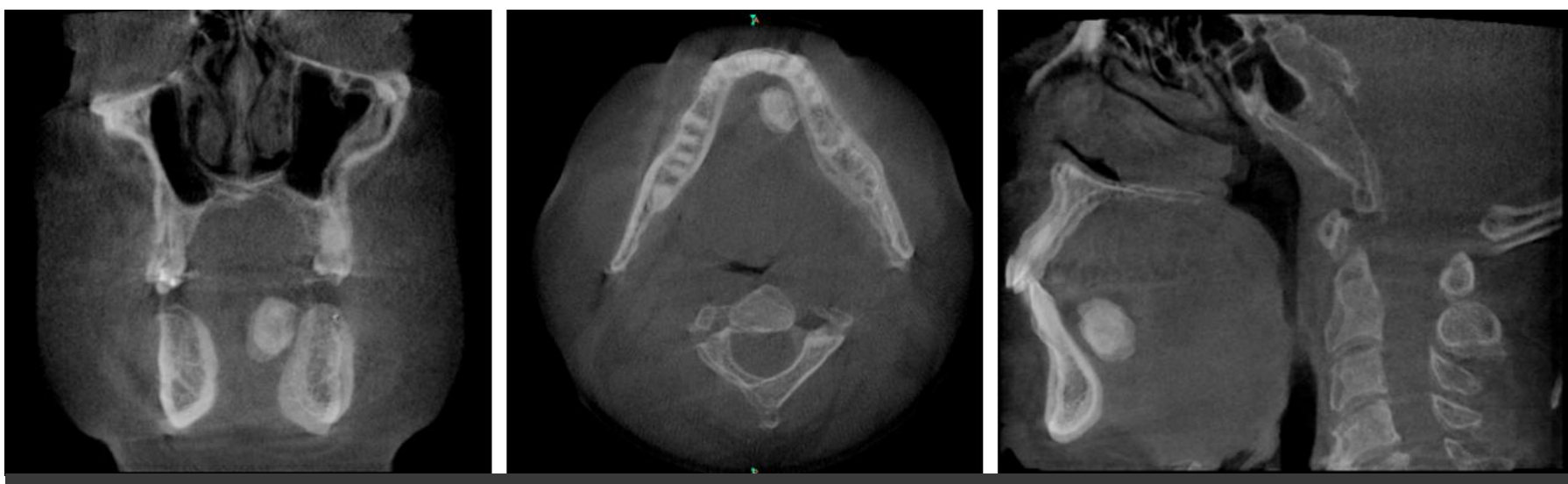

Figura 3. Imagen tomográfica donde se puede apreciar la localización y el tamaño del sialolito.

se presentaba inflamada dura y no dolorosa a la palpación. La evaluación imagenológica se inicia con la realización de una radiografía panorámica en la que se pudo evidenciar una imagen radiopaca de bordes definidos redondeada de aproximadamente $2,5 \mathrm{~cm}$ de diámetro en al piso de la boca del lado izquierdo que abarcaba desde el incisivo lateral hasta distal del segundo premolar (Figura 2), posteriormente se realizó un estudio tomográfico (cone beam) con lo que se pudo evidenciar la longitud y localización de la lesión (Figura 3).

En base a los hallazgos clínicos e imagenológicos, se describió un diagnóstico de sialolito del
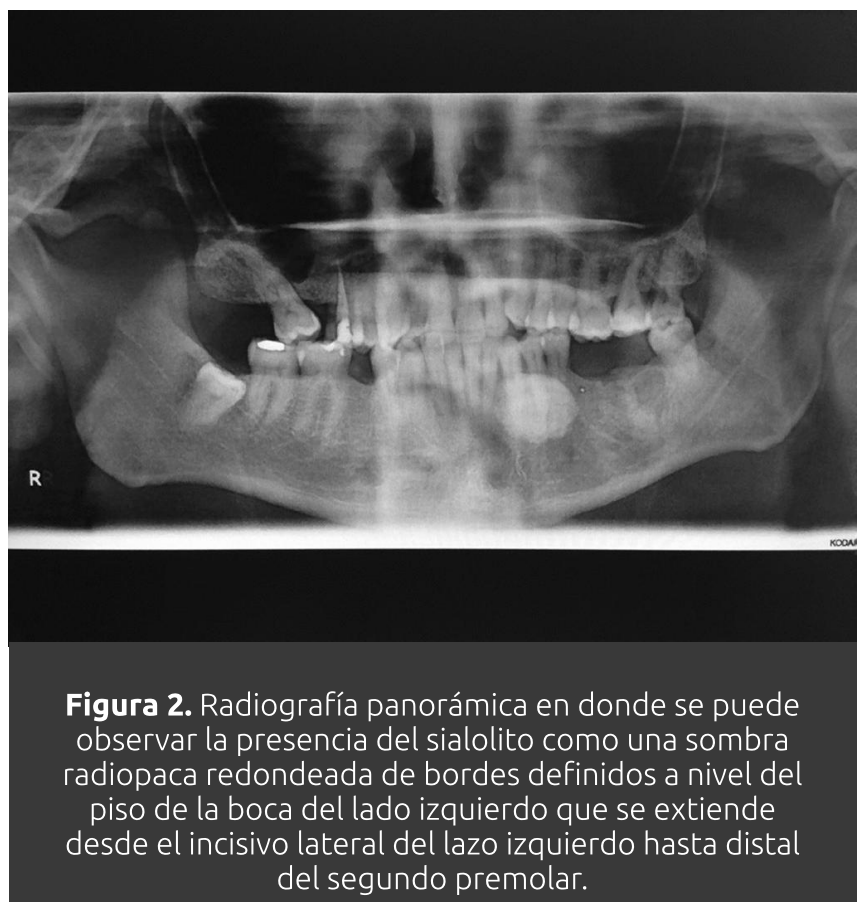

conducto submandibular izquierdo. Se optó por la remoción quirúrgica del sialolito bajo anestesia local (Figura 4,5). El sialolito removido tenía una medida de 2,5 cm de diámetro (Figura 6).

\section{Discusión}

La etiología exacta y la patogenicidad de los cálculos salivales es aún desconocida, el origen del cálculo radica en un estancamiento intermitente de saliva lo cual produce un cambio en el elemento mucoide de la misma que forma un gel, el cual proporciona un ambiente idóneo para la deposición de sales y otras sustancias orgánicas formando un cálculo 1,3. De acuerdo con la literatura los sialolitos usualmente tienen un tamaño de $1 \mathrm{~mm}$ o a menos de $1 \mathrm{~cm}$, en raras ocasiones pueden llegar a medir más de 1,5cm; el tamaño promedio reportado por Vandana \& cols. es de $6-9 \mathrm{~mm}$. Además existe una clasificación de sialolitos gigantes los cuales para entrar en esta categoría tiene que sobrepasar los $15 \mathrm{~mm}$ en cualquiera de sus dimensiones o $1 \mathrm{~g}$ en peso $\mathbf{2 , 7 , 8 , 3}$.

Los síntomas más comunes de sialolitos son dolor recurrente e inflamación de la glándula durante la alimentación, pero en ocasiones la sialolitiasis puede ser completamente asintomática como en el caso presentado en el cual el paciente no refiere ninguna molestia ni sintomatología y se detecta la presencia del sialolito por un examen radiográfico rutinario. ${ }^{2,6,9,8}$ El cálculo también puede causar obstrucción de saliva favoreciendo 


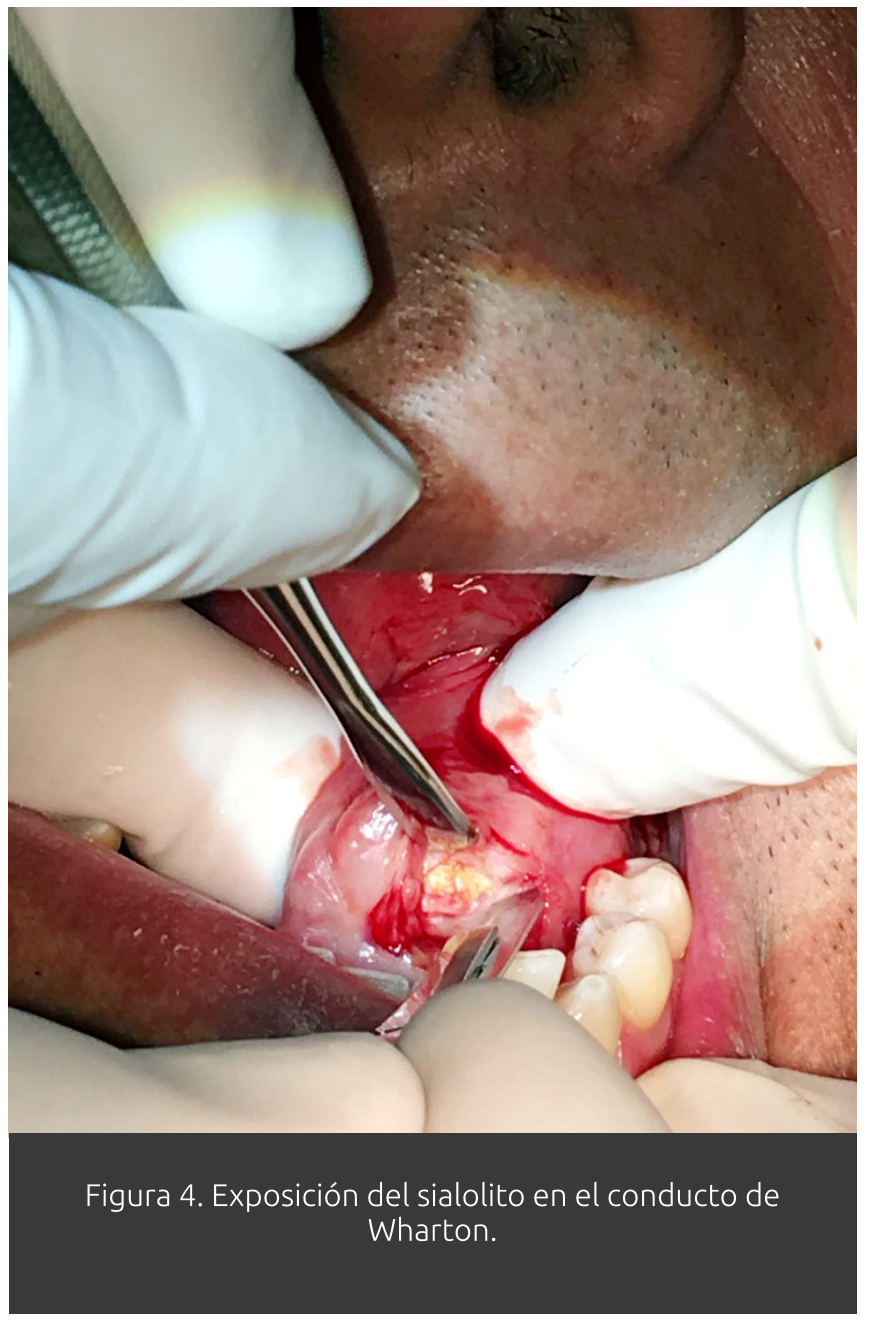

el asentamiento de las bacterias dentro del parénquima glandular lo que puede dar lugar a infecciones, estas infecciones pueden ser recurrentes y causar fibrosis ${ }^{1}$. Los sialolitos están constituidos en un $82 \%$ material inorgánico y 18\% de material orgánico; el material orgánico está compuesto de varios carbohidratos y aminoácidos mientras que el material inorgánico se encuentra compuesto de fosfato cálcico con pequeñas cantidades de carbonato en forma de hidroxiapatita, además de magnesio, potasio y amonio ${ }^{\mathbf{9}, 6}$.

En nuestro caso la gandula afectada fue la submandibular, lo que según Omezli, Singh, Oliveira, Khaladkar, entre otros, es la glándula más afectada con un $80-90 \%$, seguida de la glándula parótida en un 5-20\%, esto se explica debido a la que la saliva de la glándula submandibular es más alcalina, tiene una concentración más elevada de calcio y fosfato y

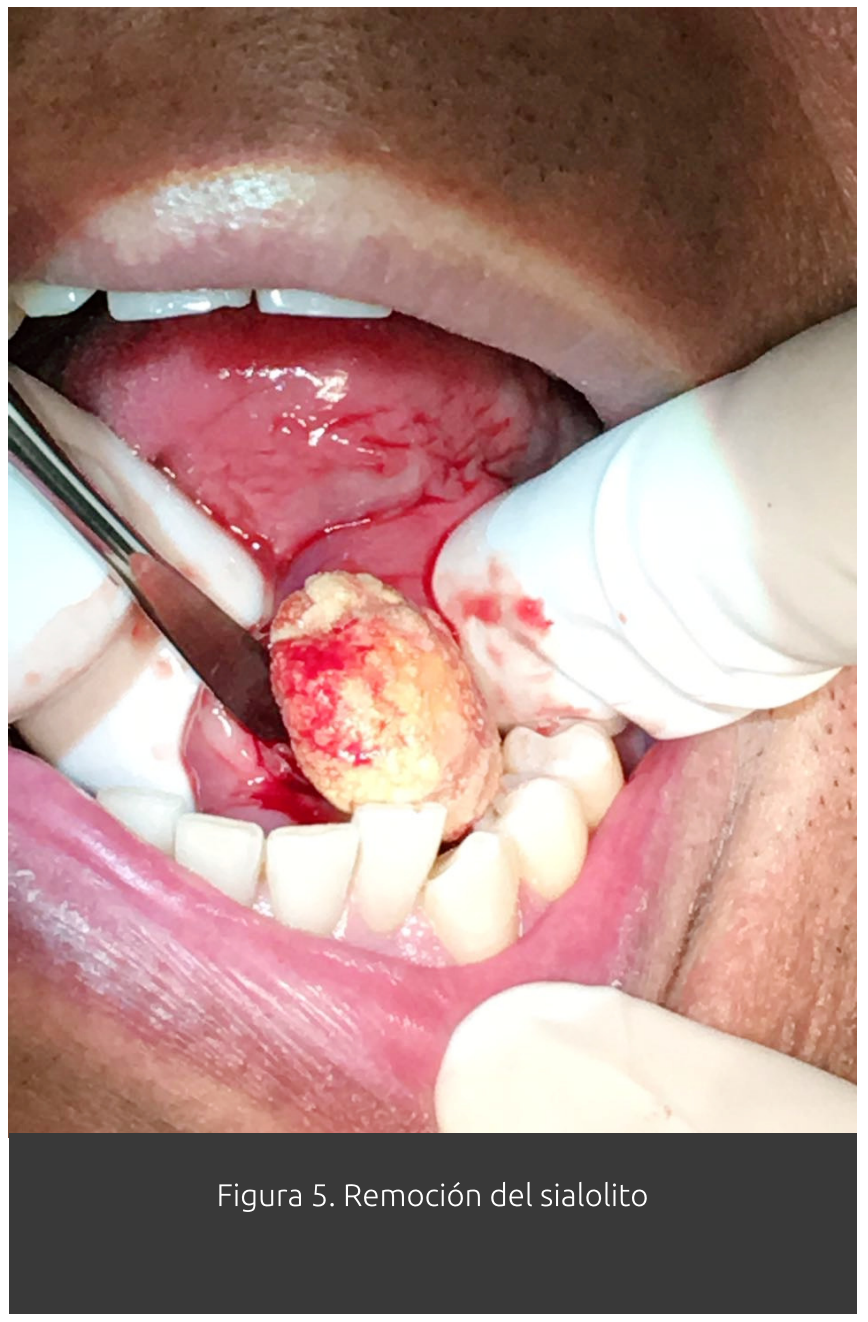

presenta una alta concentración mucosa en comparación con la glándula parótida y sublingual, el conducto es más largo y ancho que el conducto de Stenon y el flujo salival va en contra de la gravedad $\mathbf{3 , 2 , 7}$. Además esta patología se puede presentar a cualquier edad, aunque frecuentemente se observan en la tercera y cuarta década de la vida, con una predilección por el sexo masculino de 2:1 según los reportes encontrados en la literatura acerca de esta patología 9 .

Clínicamente se presenta como un nódulo submucoso asintomático único, pequeño y redondo el cual es firme y se mueve libremente en el tejido. Entre los estudios previos que se pueden realizar se encuentran las radiografías presentándose como una sombra radiopaca homogénea bien definida redonda u ovalada, pocos cálculos pueden presentarse radiolúcidos lo que podría dificultar su valoración por medio 


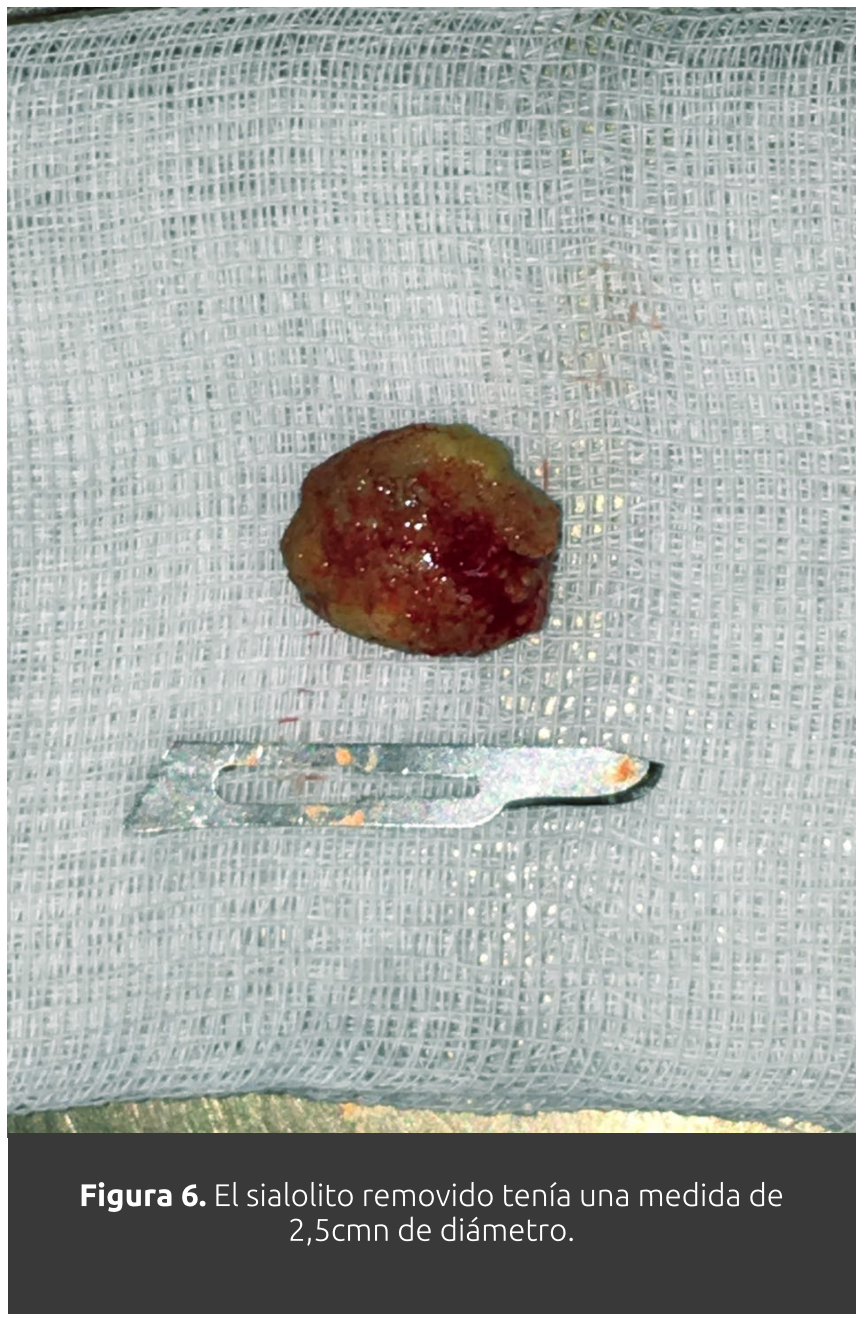

de este estudio; estudios diagnósticos más precisos son la tomografía y la ultrasonografía 10,9 .

El tratamiento para los sialolitos gigantes así como para los de tamaño regular, es la restauración de la secreción de la saliva normal, esto se puede lograr por medio de tres vías remoción a través de la cavidad oral, sialoendoscopía y resección de la glándula 7,3,2. El tratamiento que se elija depende del sitio, tamaño, forma y numero. Usualmente el tratamiento de primera elección es la remoción completa del Sialolito a través de la cavidad oral que es la manera menos invasiva, este fue el tratamiento elegido en este caso, presentando una evolución favorable sin signos de recidivas ni alteración de las estructuras ni las funciones de la zona ${ }^{10,7}$. La remoción quirúrgica de la glándula está indicada en las situaciones en las que la apertura del conducto creado provoca infecciones recurrentes de la glándula debido a la entrada de fluidos orales ${ }^{2}$.

La tasa de recidiva de esta lesión es del 18\% y cuando lo hacen pueden producir la destrucción del parénquima de la glándula provocando una sialoadenitis crónica que va a exigir la eliminación de la glándula. Actualmente en nuestro país no existen datos epidemiológicos de la prevalencia ni recidiva de esta patología, por lo que sería recomendable obtener estas cifras, para determinar si se concuerda con las estadísticas internacionales o hay variaciones en relación a estas.

\section{Conclusiones}

En la actualidad existen varios métodos para el tratamiento de los sialolitos, dependiendo de la glándula afectada y la localización de este. La remoción intraoral constituye el tratamiento de elección para la remoción de los que se localizan en el conducto de la glándula submandibular, debido a que esta es la vía menos invasiva para su remoción, además, los pacientes deben tener un seguimiento regular para controlar que no se produzcan recidivas.

\section{Referencias bibliográficas}

1. Gadve, V., Mohite, A., Bang, K., Shenoi, S. Unusual giant sialolith of Wharton's duct. Indian journal of dentistry. 2016; 7(3): 162164.

2. Singh, R., Dhawan, A., Bhullar, K., Malhotra, S. Giant submandibular gland duct sialolith mimicking an impacted canine tooth. National journal of maxillofacial surgery. 2015; 6(1): 89-92.

3. Omezli, M., Ayranci, F., Sadik, E., Polat, M. Case report of giant sialolith (megalith) of the Wharton's duct. Nigerian journal of clinical practice. 2016; 19(3): 414-417. 
4. Oliveira, T., Fernandes, I., Paez, E., Ferreira, R., Minenti, P. Giant sialolith of submandibular gland duct treated by excision and ductal repair: Case report. Brazilian Journal of Otorhinolaryngology. 2016; 82(1): 112-115.

5. Debnath, S., Adhyapok, A. Sialolithiasis of an accessory parotid gland: an unusual case. British journal of oral and maxillofacial surgery. 2015; 537(7): 658659.

6. Schroder, S., Andersson, M., Wohlfahrt, J., Wagner, N., Bardow, A., Homoe, P. Incidence of sialolithiasis in Denmark: a nationwide population-based register study. European archives of otorhinolaryngology and head \& neck. 2016.

7. Iqbal, A., Gupta, A., Natu, S., Gupta, A. Unusually large sialolith of Wharton's duct. Annals of maxillofacial surgery. 2012; 2(1): 70-73.

8. Sunder, V., Chakravarthy, C., Mikkilinine, R., Mahoorkar, S. Multiple bilateral submandibular gland sialolithiasis. Nigerian journal of clinical practice. 2014; 17(1): 115-118.

9. Khaladkar, S., Das, G. Sialolithiasis in remnant of Wharton's duct: A case report with radiologival review. Pakistan journal of radiology. 2016; 26(3): 246-248.

10. Kimura, M., Enomoto, A., Shibata, A., Nishwaki, S., Umemura, M. A Case of Sialolithiasis in a Minor Salivary Gland of the Buccal Mucosa. Journal of clinical and diagnostic research. 2016; 10(11): ZD06ZD07. 